\title{
VORWORT DES AUTORS ZUR DEUTSCHEN AUSGABE
}

Die Programmiersprache PASCAL ist das Resultat von langjährigen Entwicklungsarbeiten, die zuerst im Rahmen der IFIP-Arbeitsgruppe 2.1 stattfanden und das Ziel hatten, einen Nachfolger für Algol 60 zu definieren. Als Vorläufer kann die Sprache Algol W betrachtet werden [1], die in den Jahren 1965-68 in Stanford implementiert wurde. Diese Arbeiten haben die Grundkonzeption von Pascal entscheidend beeinflußt [2]. Sie erbrachten die Erkenntnis, daß eine moderne Sprache unbedingt auch zum Bau komplexer SoftwareSysteme wie Compiler selbst verwendbar sein müsse. Die bescheidenen zur Verfügung stehenden Arbeitskräfte und Mittel zwangen jedoch stets, den Umfang der Sprache möglichst klein zu halten. Der daraus resultierende, günstige Kompromiß zwischen weiter Verwendbarkeit und verhältnismäßig geringem Aufwand zur Implementation dürfte vor allem für den späteren Erfolg der Sprache verantwortlich sein [3].

Die Sprache findet dank ihrem systematischen Aufbau heute vor allem im Programmierunterricht Verwendung. Sie gestattet die rasche Einführung in die Grundkonzepte nach modernen Methoden des strukturierten Programmierens $[4,5,6]$ und erlaubt danach das direkte Vordringen $\mathrm{zu}$ fortgeschrittenen Konzepten der Datenstrukturierung [7]. Auf wissenschaftlichem Gebiet hat Pascal zu neuen Arbeiten auf den Gebieten der mathematischen Sprachdefinition [8] und der Multiprogrammierung [9] geführt, indem sie entsprechend erweitert wurde.

Ich möchte meiner Genugtuung Ausdruck geben, daß nun auch eine deutsche Ubersetzung der 1972 leicht revidierten Definition von Pascal verfügbar wird, und möchte dafür den Übersetzern, dem Akademie-Verlag und seinen Herausgebern bestens danken.

N. WIRTH

[1] N. Wirth und C. A. R. Hoare, ,A Contribution to the Development of Algol", Comm. ACM 9, 6, 413-432 (Juni 1966).

[2] N. WrRTh, ,,The programming language Pascal“, Acta Informatica 1, 35-63 (1971).

[3] -, ,The design of a Pascal compiler", Software - Practice and Experience 1, 309-333 (1971).

[4] -, ,Systematisches Programmieren“, Teubner-Verlag, Stuttgart (1973).

[5] K. JENSEN and N. WIRTH, „Pascal - User Manual and Report", Lecture Notes in Computer Science, Vol. 18 (1974), and Springer Study Edition (1975), both SpringerVerlag. 
[6] H. Schauer, „Pascal für Anfänger“, R. Oldenbourg-Verlag, München (1975).

[7] N. WiRTH, ,Algorithmen und Datenstrukturen“, Teubner-Verlag, Stuttgart (1975).

[8] C. A. R. HOARE and N. WIRTH, ,,An axiomatic definition of the programming language Pascal“, Acta Informatica 2, 335-355 (1973).

[9] P. Brinch Hansen, ,The Programming Language Concurrent Pascal“, IEEE Trans. on Software Engineering 1, 2, 199-206 (June 1975). 